\title{
ENSAIOS METODOLÓGICOS EM ANTROPOLOGIA ENGAJADA
}

JOÃO VITOR DE FREITAS MOREIRA ${ }^{1}$

$U F M G, B R A S I L$

\section{RESENHA}

KIRSCH, Stuart. Engaged Anthropology. Oakland (California): University of California Press. 2018, 306p.

Stuart Kirsch é um professor no departamento de antropologia da Universidade de Michigan. Desenvolveu pesquisas em Papua-Nova Guiné, uma das mais tradicionais regiões etnográficas do mundo. Tem mais de duas décadas de trabalho de campo com imersões prolongadas, típicas dos trabalhos antropológicos, e uma grande quantidade de dados etnográficos. O que se espera, aos moldes de autores que lá estiveram como Marilyn Strathern e Roy Wagner, são análises acadêmicas das mais apuradas, com importantes insights adquiridos a partir da autoridade que um etnógrafo, na prática etnológica, detém. Entretanto, esse não é o caso de Stuart Kirsch. Não é devido ao fato de seus estudos e análises não gozarem de alto requinte e estarem ao nível de seus predecessores. 0 motivo é outro: o modo como seu trabalho de campo é desenvolvido.

Kirsch, que conduziu pesquisas iniciais entre os Yonggom na década de 1980, tornou-se um antropólogo engajado por acidente. Não tinha a pretensão de desenvolver uma forma de pesquisa antropológica engajada, ou que apresentasse qualquer consideração política. Porém, após dois anos de pesquisas, os Yonggom pediram sua ajuda para abordar os problemas ambientas causados pela Ok Tedi mine. A partir daí se percebeu envolvido como ator político nas lutas contra a poluição e o desastre ambiental deixados pela OK Tedi mine. Endereçando argumentos em jornais locais, participando de campanhas, ofertando depoimentos judiciais e praticando advocacy nas terras de Papua-Nova Guiné (e fora delas), entendeu como sua participação no caso influenciou sua pesquisa

\footnotetext{
${ }^{1}$ Mestrando em Antropologia do Direito (Universidade Federal de Minas Gerais, Brasil). E-mail: joaovitorfmoreira@gmail.com
} 
e textos. Especificamente, publicou em 2014 um importante trabalho no qual faz o diagnóstico do comportamento coorporativo das empresas de mineração na relação com a crítica, ressaltando as estratégias adotadas pelo empreendimento da Ok Tedi mine (ironicamente pertencente à BHP Billiton) na tentativa de neutralizar o desastre e continuar externalizando os custos de produção.

Desde esse tempo, o que podemos encontrar nas análises e textos de Stuart Kirsch é a caracterização de uma pesquisa engajada cuja interferência "expand the possibilities of the discipline within and beyond academy" (KIRSCH, 2018, p. 46). Mas este livro de Kirsch segue um caminho diferente das reflexões sobre o campo que muitos autores e autoras realizam após alcançarem certa estabilidade acadêmica. Ao invés de apresentar reflexões gerais, ele oferta o modo como suas experiências enquanto etnógrafo e expert possibilitaram estar mais próximos dos enquadramentos de pesquisa que as práticas tradicionais da thick discription (GEERTZ, 1973) tanto requerem.

Contudo, a pergunta que surge nesse ponto é: o que se compreende como antropologia engajada? Ou seus termos assemelhados como antropologia ativista, antropologia colaborativa, antropologia militante e antropologia pública? Um termo que talvez nos remeta a Terrence Turner e seu trabalho com os Kayapó - com destaque para a polêmica disputa contra Napoleon Chagnon (GRAHAM, 2003, LIMA et al., 2008) - e Bruce Albert (1997). A ideia de antropologia engajada é reconectada a uma matriz teórica por Stuart Kirsch, que afirma entender a ligação do tipo de pesquisa etnográfica que pratica às críticas do movimento writing cultures, mas observa que: "the writing cultures debates addressed the question of reflexivity within the text, including the influence of the author's political commitments and positionality on ethnography. In contrast, engaged anthropology is concerned with reflexivity beyond the text [...]" (KIRSCH, 2018, p. 2).

Mas isso não é algo que somente encontramos em Kirsch. Em 2008, por exemplo, foi realizado um Wenner-Gren Workshoop sobre antropologia engajada, publicado como um número na Current Anthropology (LOW; MERRY, 2010), que coloca em questão o núcleo da ideia de engajamento (também entendido como envolvimento) nas pesquisas antropológicas. $E$ é justamente isso que Kirsch busca evidenciar nesse livro. Trata-se, portanto, de uma política de participação e não de representação, uma ideia que nosso autor diz ser uma suplementação das formas convencionais de pesquisa etnográfica.

Isso significa, em termos diretos, uma responsabilidade com o enquadramento de pesquisas e um compromisso em mobilizar a antropologia para intervenções construtivas na política (KIRSCH, 2010, 2018). Antropologia engajada, mais do que simplesmente endossar uma causa, ou trazer à atenção para um descontentamento particular, é um projeto de prática etnográfica que se modula às condições requeridas por aqueles que se estudam: "They [engaged anthropologists] seek nothing less than the enrichment of the finest traditions of ethnographic research 
while simultaneously addressing important questions of social justice" (KIRSCH, 2010, p.78).

Mas como pode o antropólogo estar vinculado às práticas engajadas e ao mesmo tempo produzir uma pesquisa etnográfica "boa o suficiente"? Não seria ainda a metáfora do "barco se afastando numa ilha de corais...o isolamento do mundo exterior" adequada para enquadrar a objetividade requerida? Talvez, o que estejamos observando é uma atualização do método antropológico, pois os povos que eles [os antropólogos] tradicionalmente estudaram agora se tornam autores da sua própria história (ALBERT, 1997), requerendo uma reciprocidade muito mais aguçada do que simplesmente distribuir uma boa centena de páginas aqui e acolá.

Ao longo dos 7 capítulos dessa obra, Kirsch nos oferta diferente exemplos etnográficos, alguns mais robustos do que outros, mas que exemplificam o modo de atuação antropológica desenvolvida por ele. Revelando, inclusive, dados e alguns pontos que, ao tempo dos eventos em que estava envolvido, poderiam ter causado transtornos e controvérsias entre o povo afetado, o que ele chama de recusa etnográfica (ethnographic refusal).

No Capítulo 1, "How political commitments influence research", Kirsch descreve sua participação no processo contra os donos da Ok Tedi Mine, apresentando um backstage que em outras publicações não foi levado em conta (KIRSCH, 2002, 2014). Ao reconhecer que sua participação nos eventos contra a Ok Tedi Mine modelou suas conclusões (o que ataca diretamente aquele fantasma positivista que nos persegue), afirma que isso também o permitiu despertar uma sensibilidade acerca da degradação ambiental e de sua afetação direta às pessoas rio abaixo. Sua participação no caso da $O k$ Tedi mais facilitou sua interação com " $k e y$ players in the conflict. [...]. Advocacy rather than neutrality allowed me to participate in, and contribute to, the struggle against the environmental problems caused by the mining project" (KIRSCH, 2018, p. 33). O que chama a atenção nesse capítulo, além de uma perspectiva apresentada pelo autor que certo observador neutro não teria, é que Kirsch coloca em questão os riscos que os antropólogos que produzem evidências para casos judicializados podem sofrer. Como a intimação a apresentar as notas de campo, bem como a participação em procedimentos legais afeta diretamente a relação com os informantes, que impedem o etnógrafo de conduzir pesquisa em outros tópicos, haja vista que ele se torna um informante dos informantes.

No capítulo 2, "When contributions are elusive", nos deslocamos dos assuntos da Ok Tedi mine para os refugiados nas fronteiras com Papua Ocidental. $\mathrm{O}$ autor inicia uma abordagem sobre o tipo de trabalho que ele fez com o outro lado (ocidental) de Papua. Especificamente, tudo se iniciou com os refugiados que se encontravam na fronteira de PNG, onde Kirsch estava conduzindo etnografia por 18 meses. Kirsch narra um ritual que presenciava entre os refugiados quando dois deles questionaram sua participação na cerimônia de iniciação "asking me what I intended to do 
with the knowledge I had gained: Would I use it to help the refugees or assist their enemies?" (KIRSCH, 2018, p. 50). Contudo, a atuação de Kirsch requerida pelo desafio posto pelos refugiados se resumiu, inicialmente, a escrever sobre a história da região de fronteira e a vida do campo de refugiados, já que o lado ocidental de Papua apresentava, e ainda apresenta, inúmeras resistências a trabalhos etnográficos. Apenas a mudança de foco dos movimentos sociais de resistência permitiu uma atuação de Krisch, momento este em que ele se sentiu confortável para atuar com lideranças políticas e ativistas da Papua Ocidental em diferentes Workshops e ONGs. Entretanto, diferente do caso narrado no Cap. 1, "I was unable to contribute much of substance or even strategy at these meetings" (KIRSCH, 2018, p. 75).

Após mais de duas décadas passadas com os refugiados da Papua Ocidental, Kirsch foi capaz de cruzar a fronteira em 2014, entrevistando uma série de pessoas entre Jayapura e Biak. Contudo, conclui que não é sempre possível a um antropólogo realizar contribuições significativas para as lutas políticas das pessoas com quem ele trabalha, questionandose sobre a possibilidade de uma antropologia engajada em áreas onde o acesso é proibido ou restrito, como em Papua Ocidental.

No Capítulo 3, "The search for alternative outcomes", Kirsch retrata sua experiência no Lakekamu River durante o verão de 1994, quando se juntou a biólogos conservacionistas que buscavam instalar um programa integrado de conservação e desenvolvimento. O objetivo era etnografar os quatro diferentes grupos linguísticos da bacia do Lakekamu (Biaru, Kurija, Kamea e Kovio). A falha na implementação do projeto levou o autor a refletir sobre as diferentes perspectivas em Papua-Nova Guiné acerca das demandas por terra e as diferenças entre os grupos que vivem na bacia do Lakekamu. Contudo, a observação mais importante da antropologia engajada no caso narrado, e que talvez funcione como uma certa limitação metodológica, é que a busca por implicações alternativas [implementação de um projeto integrado de conservação e desenvolvimento] pode influenciar os resultados das análises. Por mais que nosso autor considere que seus dados etnográficos ainda são consistentes, ressalta que o efeito de sua atuação na bacia do rio Lakekamu sugeriu a necessidade de maior atenção a questão da conservação e modificou sua atuação em relação à mineração.

É importante, nesse ponto, dizer que os dados e exemplos que Kirsch traz nesse livro são dispostos em ordem não cronológica. Sua atuação direta contra a $O k$ Tedi mine se intensificou após sua atuação na bacia do Lakekamu, ao passo que sua atuação etnográfica na borda de Papua Ocidental se inicia em 1989 e prolonga-se até o cruzar fronteiras em 2014. Todas as três experiências etnográficas, contudo, são desenvolvidas em Papua. No capítulo 4 as coisas mudam. "When the intervention fails, does the research still matter?" aborda uma atuação frustrada nas Ilhas Salomão, que se sucedeu devido a um convite do escritório de direito que atuava no caso da Ok Tedi mine e, nos anos de 1998, buscava base para acionar a Gold Ridge mine legalmente. Kirsch 
tinha a função de coletar dados sobre as posses de terra e sobre os direitos de propriedade afetados pela mineração, bem como de documentar o impacto ambiental e social do projeto.

Entretanto, as tensões nas ilhas Salomão resultaram em um conflito civil que paralisou o país de 1998-2003. A pergunta que Kirsch busca responder nesse capítulo é: O dado etnográfico coletado no contexto de antropologia engajadas ainda teria valor quando as circunstâncias mudam e o projeto de atuação muda sua razão de ser?

O capítulo 5 traz mais um exemplo fora das terras de Papua NovaGuiné, buscando investigar "How analysis of local contexts can have global significance". O objetivo era produzir contribuições a um caso levado ao Tribunal estabelecido em 1999 nas Ilhas Marshal para julgar a interferências, destruições e compensações necessárias decorrentes dos efeitos de uma bomba de hidrogênio detonada pelos Estados Unidos em 1954. Os insights aqui providos são bastante importantes nas reflexões acerca da perda cultural e dos direitos de propriedade cultural. Essa abordagem, que enfatiza a perda mais do que a mudança, assume contornos devido ao discurso dos povos indígenas afetados que incorporam essa vertente da cultural loss (KIRSCH, 2018, p. 138). A noção de perda cultural acionada no caso tenciona as práticas tradicionais da antropologia, mas Kirsch acredita que ela "can be used to adress this critical blind spot of culture concept, in which loss is unseen or undervalued" (2018, p. 139).

Os capítulos 6 e 7 relatam perspectivas distantes das terras do extremo oriente do globo. Kirsch nos situa em "The risk of intervention" no inverno de 2010, no qual foi convidado para participar de reuniões sobre native americans justamente por ser ele, na Universidade de Michgan, um representante da relação entre etnografia e ativismo. 0 capítulo, totalmente distinto dos outros - e que coloca em questão seu papel diante dos demais capítulos -, aborda uma controvérsia que uma fala sua gerou entre os estudantes de arqueologia ao afirmar que "we're not here to burn down the museum [...] even if some of us would like to see that happen" (KIRSCH, 2018, p. 178). Subsequentemente, o autor menciona uma carta feita pelos alunos de arqueologia em repúdio à fala, que acabou circulando até chegar ao vice-presidente para pesquisa da universidade. Bom, poderíamos esperar uma conclusão sobre esse episódio, como nos demais capítulos. Poderíamos esperar uma análise de como é a repercussão no campus de uma universidade a que se é vinculado quando se pratica a antropologia engajada. Entretanto, nada disso aparece nesse curto capítulo 6. Kirsch termina com: "It is not difficult to imagine a different outcome for a sociocultural anthropologist in a department dominated by archaeologists, a university in which democratic decision-making on tenure cases is not the norm [...]' (KIRSCH, 2018, p. 179) 
Por fim, adentramos ao último capítulo denominado "Dilemas of an expert witness". O autor apresenta dois affidavits ${ }^{2}$ escritos para a Comissão Interamericana de Direito Humanos ${ }^{3}$. O primeiro está relacionado à recusa do Estado de Suriname em reconhecer os direitos territoriais indígenas, apesar da obrigatoriedade legal interna. O segundo, traz uma abordagem acerca dos problemas de títulos de terra indígena na Guyana. Kirsch reflete sobre as investidas etnográfica de curto tempo feita por um expert, concluindo acerca do primeiro caso, no Suriname, que os povos Lokono e Kalina requerem controle coletivo de seus territórios e recursos, afirmando que necessitam ser livres em seus próprios territórios. Reporta a falha do Estado em respeitar o direito territorial indígena na reserva natural de Galibi, bem como evidencia o modo como a mineração de bauxita afeta a reserva natural de Wane Kreek. Tais pontos, associados com a falta de reconhecimento pelo Estado dos direitos indígenas, deixam os indígenas sem qualquer influência ou recurso legal para demandar da BHP Billiton e da Suralco o ressarcimento e a limpeza dos prejuízos causado pela mineração.

Por outro lado, o segundo caso relata conflitos por terra entre o povo Akawaio de Isseneru e o Estado da Guyana, especialmente após a redução de um quarto das terras feitas pelo então ministro de assuntos indígenas. Kirsch demonstra como a Guyana faz uso retórico para afirmar um discurso de não-indígenas sobre os Akawaio, argumentando que estes teriam perdido sua "tradicionalidade" em virtude de casamentos interétinicos e por serem falantes de inglês - uma política de estado não muito distante de alguns países vizinhos. Kirsch, todavia, consegue evidenciar que "land is very important to the Akawaio people: they describe the forest as their grocery, their doctor's office, and their university" (2018, p. 2004). Nesse ponto, o discurso sobre a não afetação da identidade se torna forte entre os Akawaio: ressaltando o contraste com a ênfase na propriedade privada nos sistemas de common e civil law, a perspectiva Akwaio entende que a terra é propriedade coletiva.

Em ambos os casos, questões sobre a mineração atravessavam o caminho dos estudos. Apesar disso, podemos considerar o conhecimento de que quando um antropólogo atua como um expert, ou é convidado a se envolver em questões eminentemente políticas, a atuação acadêmica se modula - ora apresentado pareceres em linguagem transversal aos advogados e comunidade, ora refutando dados etnográficos para evitar confrontos ou conflitos internos. Em ambos os casos Kirsch "ernaed their trust, so that they consented to my request to continue writing about the situation beyond the affidavit, despite their criticism of antropologists who have written about them in the past" (2018, p. 220).

\footnotetext{
${ }^{2}$ Depoimento juramentado de um expert feito por escrito e normalmente apresentado em procedimentos legais.

3 A Comissão e a Corte Interamericana de Direitos Humanos são dois órgãos que integram o Sistema Interamericano de Direitos Humanos criados no bojo da Organização dos Estados Americanos (OEA). A função do Sistema Interamericano está disposta na Carta da OEA e tem como objetivo principal promover, fiscalizar e defender os Direitos Humanos em relação aos 35 países membros.
} 
Ao fim e ao cabo, temos um exemplo interessante de um experimento em antropologia engajada, mas sem muitas delimitações acerca do como, apresentando-se práticas de um antropólogo e sua atuação específica, cuja nervura central tem como foco as demandas judiciais. Se é verdade que "the anthropologist's 'observation' is no longer merely 'participant'; his social 'participation' has become both the condition and the framework of his field research" (ALBERT, 1997 p. 50), Stuart Kirsch se monstra um exemplo de tal prática de envolvimento ou engajamento. É certo, contudo, que alguns de seus dados, especificamente os relacionados à mineração em Papua-Nova Guiné, ganham maior força do que outros, dado o prolongado período de tempo do engajamento. Isso não significa que as demais atuações apresentadas não tenham respaldo etnográfico ou etnológico.

A grande conclusão que podemos alcançar desse tipo de pesquisa em antropologia é que os antropólogos engajados certamente têm acesso aos seus interlocutores de forma diferenciada, pois suas contribuições aos projetos, casos e episódios permitem oportunidades de interação muito maior do que as métricas de pesquisa positivista. Além disso, a interação com os interlocutores se expande. Assim, não se resume somente ao povo que estuda, mas contempla advogados, executivos, ONGs, representantes de governo e além do acesso a espaços de adjudicação dos mais distintos. Entretanto, o método empregado em campo sempre dependerá das demandas postas ao antropólogo engajado, o que o tornará mais vulnerável às críticas internas ao campo. Isso, certamente, atrairá impasses, mas o que não podemos negar é que os resultados de pesquisas que Stuart Kirsch nos apresenta estão beyond text e interferem na política local, estatal e internacional. 


\section{Referências bibliográficas}

ALBERT, B. 'Ethnographic Situation' and Ethic Movements: notes on postMalinowskian fieldwork. Crítique of Anthropology, n. 1, v. 17, 1997, p. 53-65.

GEERTZ, C. Interpretation of Cultures: Selected essays. New York (NY): Basic Books, 1973.

GRAHAM, L R. How Should an Indian Speak? Amazonian Indians and the Symbolic Politics of Language in the Global Public Sphere. In: WARREN, Kay B.; JACKSON, Jean E. (Ed.) Indigenous Movements, Self Representation, and the State in Latin America. Austin: University of Austin Press, 2003, p. 181-228.

KIRSCH, S. Anthropology and Advocacy: a case study of the campaign against the Ok Tedi Mine. Critique of Anthropology, n. 2, v. 22, 2002.

KIRSCH, S. Experiments in engaged anthropology. Collaborative Anthropologies, v. 3, p. 69-80, 2010.

KIRSCH, S. Mining Capitalism: The relationship between corporations and their critics. Oakland (CA): University of California Press, 2014.

KIRSCH, S. Engaged Anthropology. Oakland (California): University of California Press. 2018, 306 p.

LIMA, E et al. Uma Antropologia Engajada: entrevista com Terence Turner. Campos, $\mathrm{n}$. 9 , v. 2, 2008, p. 139-157.

LOW, S M.; MERRY, S. E. Engaged anthropology: Diversity and Dilemmas. Current Anthropology, v. 51, suplementação 2, out. 2010.

Recebido em: 24/01/2019* Aprovado em: 18/05/2019* Publicado em: 30/12/2019 